\title{
Preliminary experience of the estimation of cerebral perfusion pressure using transcranial Doppler ultrasonography
}

\begin{abstract}
E A Schmidt, M Czosnyka, I Gooskens, S K Piechnik, B F Matta, P C Whitfield,
\end{abstract} J D Pickard

\begin{abstract}
Objective-The direct calculation of cerebral perfusion pressure (CPP) as the difference between mean arterial pressure and intracranial pressure (ICP) produces a number which does not always adequately describe conditions for brain perfusion. A non-invasive method of CPP measurement has previously been reported based on waveform analysis of blood flow velocity measured in the middle cerebral artery (MCA) by transcranial Doppler. This study describes the results of clinical tests of the prototype bilateral transcranial Doppler based apparatus for non-invasive CPP measurement (nCPP). Methods-Twenty five consecutive, paralysed, sedated, and ventilated patients with head injury were studied. Intracranial pressure (ICP) and arterial blood pressure (ABP) were monitored continuously. The left and right MCAs were insonated daily (108 measurements) using a purpose built transcranial Doppler monitor (Neuro $Q^{\mathrm{TM}}$, Deltex Ltd, Chichester, UK) with software capable of the noninvasive estimation of CPP. Time averaged values of mean and diastolic flow velocities (FVm, FVd) and ABP were calculated. nCPP was then computed as: ABP $\times \mathbf{F V d} / \mathbf{F V m}+14$.
\end{abstract}

Results-The absolute difference between real CPP and nCPP (daily averages) was less than $10 \mathrm{~mm} \mathrm{Hg}$ in $89 \%$ of measurements and less than $13 \mathrm{~mm} \mathrm{Hg}$ in $92 \%$ of measurements. The $95 \%$ confidence range for predictors was no wider than $\pm 12 \mathrm{~mm}$ $\mathrm{Hg}(n=25)$ for the CPP, varying from 70 to $95 \mathrm{~mm} \mathrm{Hg}$. The absolute value of side to side differences in nCPP was significantly greater $(p<0.05)$ when CT based evidence of brain swelling was present and was also positively correlated $(p<0.05)$ with mean ICP.

Conclusion-The device is of potential benefit for intermittent or continuous monitoring of brain perfusion pressure in situations where the direct measurement is not available or its reliability is in question. (F Neurol Neurosurg Psychiatry 2001;70:198-204)

Keywords: cerebral perfusion pressure; transcranial Doppler; non-invasive monitoring

The concept that the difference between arterial (ABP) and intracranial pressure (ICP) represents cerebral perfusion pressure $(\mathrm{CPP})^{1}$ is well established in clinical practice. Identification of secondary brain insults ${ }^{2}$ and a clear dependence between average CPP and outcome after severe head injury, demonstrated in the 1970s and 1980s, ${ }^{3}$ have contributed to the formulation of CPP oriented therapy. ${ }^{4}$ In our centre CPP oriented management was rigorously introduced in 1995, with a critical threshold of CPP set by consensus at a target value of $70 \mathrm{~mm} \mathrm{Hg}$. CPP has ceased to be a significant predictor of outcome. ${ }^{5}$

This may provoke suspicions that the concept of $\mathrm{CPP}$ as a number $(\mathrm{CPP}=\mathrm{ABP}-\mathrm{ICP})$ is not always adequate and assessment of cerebral perfusion, based on cerebral blood flow (CBF) or brain oxygenation, would be more appropriate. Unfortunately, existing methods for the measurement of CBF are not suitable for continuous bedside monitoring. Non-invasive technology for the measurement of brain oxygenation based on near infrared spectroscopy is still unreliable ${ }^{6}$ and direct measurements are extremely localised.

Transcranial Doppler ultrasonography offers non-quantitative measurements of $\mathrm{CBF}$. Changes in global CBF can be monitored continuously and non-invasively using blood flow velocity, ${ }^{7}$ provided that the diameter of basal cerebral arteries does not change. Exploring this concept, many independent authors have emphasised that specific patterns of transcranial Doppler waveform follow the state of inadequate cerebral perfusion caused by a decrease in $\mathrm{CPP}^{8-10}$ Although converting this specific distortion of blood flow velocity waveform to CPP is difficult, ${ }^{8}{ }^{10}$ this step seems to be necessary as most therapeutically used protocols are still based on a value of CPP expressed as a number. A non-invasive method for the assessment of CPP using transcranial Doppler blood flow velocity waveform has been described previously. ${ }^{10}$ Using this methodology, the prototype bilateral transcranial Doppler machine with a built in algorithm to assess CPP using an externally measured value for $\mathrm{ABP}$ has been designed (Neuro $\mathrm{Q}{ }^{\mathrm{TM}}$ Deltex Ltd, Chichester, UK). The prototype has been used in a prospective study to address questions about the accuracy of non-invasive CPP estimation, and the significance of side to side differences in non-invasive CPP.

\section{Clinical material and methods}

PATIENTS

With the approval of the local ethics committee non-invasive CPP was assessed daily using the 
prototype device based on transcranial Doppler ultrasonograph (Neuro-Q ${ }^{\mathrm{TM}}$ ) in 25 patients with closed traumatic brain injury. Only patients with diffuse cerebral injury or with focal lesions mild enough not to require a surgical procedure were studied. The patients were admitted to the Neuroscience Critical Care Unit in Addenbrooke's Hospital, Cambridge, United Kingdom, from June to December 1998 .

There were 21 men and four women; the mean age was 36 (range 18 to 76 ).

All patients were sedated, paralysed, and ventilated to maintain adequate oxygenation and mild hypocapnia (30-35 $\mathrm{mm} \mathrm{Hg}$ of arterial blood $\mathrm{CO}_{2}$ partial pressure). Intravenous fluids and inotropic support were used to maintain a target CPP above $70 \mathrm{~mm} \mathrm{Hg}$. The ICP was kept below $25 \mathrm{~mm} \mathrm{Hg}$ by vigorous management, which was possible in all but one patient (who died).

The core temperature was kept in a normal range $\left(35^{\circ} \mathrm{C}-37^{\circ} \mathrm{C}\right)$. Cooling measures were used only to avoid hyperthermia. Active hypothermia was not pursued. Brain CT was pragmatically analysed and graded using a simplified system based on the classification of Marshall et al. ${ }^{11}$

The following radiographic features were scored:

- Disappearance of sulci

- Effacement of ventricles

- Reduction of the white-grey matter difference - that is, disappearance of the whitegrey matter difference in more than $50 \%$ of the slices.

- Basal cistern compression.

The sum of these factors produced a rudimentary swelling score from 0 to 4 .

MONITORING

In all patients systemic and cerebrovascular circulation was monitored.

Cardiovascular dynamics were routinely assessed using an ABP line (radial or dorsalis pedis arteries), central venous and pulmonary artery pressure monitoring, ECG, and pulse oximetry.

A fibreoptic transducer (Camino, Camino Lab, San Diego, CA, USA) or Codman microsensor (Codman, Randolph, MA, USA) was inserted intraparenchymally into the right frontal region and fixed to the skull with a bolt to measure ICP.

The MCA blood flow velocity was measured daily, beginning on the day of admission and continuing until the ICP bolt was removed. Special care was taken to perform the recording during a stable period of ventilation and haemodynamics (for 30 minutes minimum; periods of recording were randomly distributed throughout the day). The MCAs were bilaterally insonated using two probes mounted on an adjustable frame (Neuro $\mathrm{Q}^{\mathrm{TM}}$ - prototype, Deltex Ltd, Chichester, UK). The signal was detected from a depth varying from 4.5 to 6 $\mathrm{cm}$. Data showing obvious artefacts (abrupt and sudden modification in the recording related to arterial line flushing, loosening of cables, electrical interference, etc) were excluded.

For final analysis, recordings obtained only from sedated, paralysed patients with closed head injury, with continuous ICP and ABP measurements, with good quality bilateral MCA flow velocity recordings were included.

DATA CAPTURE AND ANALYSIS

Analogue outputs from the pressure monitors and the Neuro $Q^{\mathrm{TM}}$ unit were connected to the analog to digital converter fitted into a laptop computer. Data were sampled (sampling frequency $50 \mathrm{~Hz}$ ), digitised, and stored on the computer hard disk using software designed for waveform recording. ${ }^{10}$ Data calculated within Neuro $Q^{\mathrm{TM}}$ were downloaded to the laptop computer via an RS 232 serial interface.

Both the Neuro $\mathrm{Q}^{\mathrm{TM}}$ and computer digital time series were processed and compared. Time averaged (mean) values of ICP, ABP, and CPP (ICPm, ABPm, CPPm respectively $(\mathrm{CPPm}=\mathrm{ABPm}-\mathrm{ICPm}))$ were calculated using time integration of waveforms for $5 \mathrm{sec}-$ ond intervals.

Time averaged mean, systolic, and diastolic values of flow velocity (FVm, FVs, and FVd respectively) were calculated after careful spectral filtration to reduce the influence of artefacts or noise and averaged within the same 5 second periods.

The non-invasive CPP (nCPP) calculation was based on a formula previously published ${ }^{10}$ : $\mathrm{nCPP}=$ mean $\mathrm{ABP} \times \mathrm{FVd} / \mathrm{meanFV}+14 \mathrm{~mm} \mathrm{Hg}$

The measurements were performed separately using left and right MCA flow velocities; left-right nCPP was designated as dCPP. The arithmetic mean of left and right nCPPs was designated as nCPP.

\section{STATISTICAL ANALYSIS}

An observational $x-y$ scatterplot was used to visualise association between all 1 minute averages of measured nCPP and CPP. A BlandAltman $^{12}$ plot was presented using averaged values of measured parameters over the whole recording period $(n=108)$. Regression analysis (CPP versus nCPP) was performed for independent data samples $(n=25)$ averaged for each patient, and the $95 \%$ confidence interval for predictors was measured. Left to right differences of CPP were analyzed using values of left and right nCPP averaged over all measurements performed in individual patients with analysis of variance (ANOVA) ( $F$ test or Kruskall-Wallis test).

\section{Results}

CPP AND NCPP

One minute averages ( $n=12$ 275), daily averages $(n=108)$, and patient averages $(n=25)$ were analysed. The distributions of the absolute difference between calculated and estimated CPP is given in the table.

A 1 minute average scatterplot visualising association between CPP and $\mathrm{nCPP}$ is presented in fig $1 \mathrm{~A}$. Distribution of the absolute difference CPP-nCPP is presented in fig $1 \mathrm{~B}$; 
Per cent proportion of non-invasive estimator of CPP fitting 5, 10, and $15 \mathrm{~mm} \mathrm{Hg}$ error ranges compared with direct (invasive) CPP ( $n=127251$ minute averages)

\begin{tabular}{|c|c|c|c|c|c|c|}
\hline \multirow[b]{2}{*}{ Error } & \multicolumn{3}{|c|}{ All 1 minute averages } & \multicolumn{3}{|c|}{ Day averages } \\
\hline & $n C P P$ & Left $n C P P$ & Right $n C P P$ & $n C P P$ & Left $n C P P$ & Right $n C P P$ \\
\hline$<5 \mathrm{~mm} \mathrm{Hg}$ & 49 & 38 & 41 & 58 & 39 & 44 \\
\hline$<10 \mathrm{~mm} \mathrm{Hg}$ & 81 & 71 & 72 & 89 & 76 & 78 \\
\hline$<15 \mathrm{~mm} \mathrm{Hg}$ & 94 & 91 & 87 & 94 & 92 & 90 \\
\hline
\end{tabular}

$\mathrm{nCPP}=$ Global non-invasive $\mathrm{CPP}$; left $\mathrm{nCPP}=$ left hemisphere $\mathrm{nCPP}$; right $\mathrm{nCPP}=$ right hemisphere $\mathrm{nCPP}$

$81 \%$ of 1 minute averages of nCPP were different from direct CPP by less than $10 \mathrm{~mm} \mathrm{Hg}$.

A Bland-Altman plot is presented in fig 2 for daily averages of CPP. Day by day averaging increased the percentage of the measurements with an absolute difference nCPP-CPP less than $10 \mathrm{~mm} \mathrm{Hg}$ to $89 \%$.

Although direct ICP was measured routinely in the right hemisphere, there were no obvious differences between error distribution on the left and right side (table).
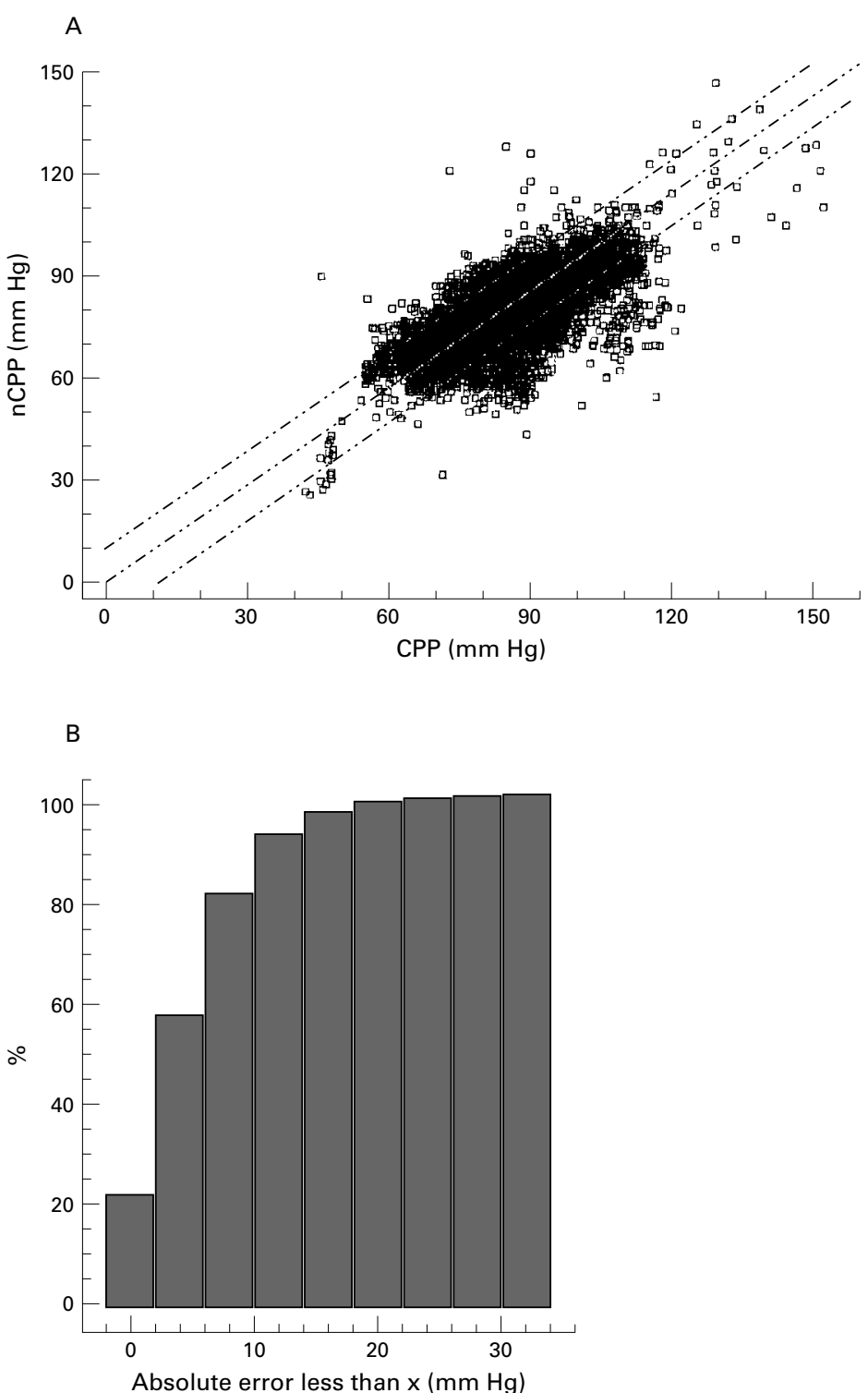

Figure 1 (A) Scatterplot of all 1 minute averages of $C P P$ ( $x$ axis) and $n C P P$ ( $y$ axis) with $x=y$ unity line and $\pm 10 \mathrm{~mm} \mathrm{Hg}$ range. (B) Cumulative histogram showing percentage of samples when the absolute difference between $n C P P$ and $C P P$ was less than a value on the $x$ axis.
Using the analysis of patient averaged data, correlation between averaged CPP and $\mathrm{nCPP}$ proved to be significant $(R=0.61 ; \mathrm{n}=25$; $\mathrm{p}=0.003)$. The lines limiting the $95 \%$ confidence region for predictors were distant from the regression line by no more than $\pm 12 \mathrm{~mm}$ $\mathrm{Hg}$ when CPP varied from 70 to $95 \mathrm{~mm} \mathrm{Hg}$.

SIDE TO SIDE DIFFERENCES IN nCPP

The mean absolute value of dCPP was 6 (SD 5.8) $\mathrm{mm} \mathrm{Hg}$. Daily observations showed that the absolute value of dCPP progressively increased with time after injury (ANOVA; $\mathrm{p}<0.012$ ). Similarly, the absolute value of the difference between left and right mean flow velocity increased with time elapsed from trauma (ANOVA; $\mathrm{p}<0.006)$. The dCPP was significantly correlated with side to side difference in mean flow velocity $(R=0.78$; $\mathrm{p}<0.0001$ ).

In patients showing midline shift on CT $(n=4)$, the estimated dCPP was significantly greater than zero (mean 12 (SD 6) $\mathrm{mm} \mathrm{Hg}$; $\mathrm{p}<0.04$, Kruskall-Wallis test, fig $3 \mathrm{~A}$ ). The swelling score was significantly associated with an increase in absolute value of dCPP $(p<0.05$, fig 3 B). Finally, an absolute value of dCPP was significantly correlated with a mean ICP (fig $3 \mathrm{C}$ ).

\section{OBSERVATIONS}

An example of 11 consecutive daily recordings in a patient with head injury is presented in fig 4. In this patient the trends in CPP and nCPP were moderately correlated in time $\left(R^{2}=0.48\right)$. The greatest discrepancy between CPP and nCPP took place on day 4 (average CPP$\mathrm{nCPP}=9 \mathrm{~mm} \mathrm{Hg}$ ), when the average ICP increased to above $40 \mathrm{~mm} \mathrm{Hg}$. This was followed by a significant and symmetric increase in blood flow velocity.

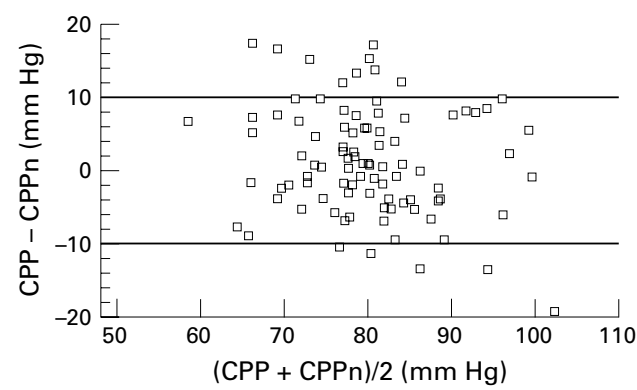

Figure 2 Bland-Altman plot of all 108 day by day measurements of $C P P=A B P-I C P$ and $n C P P$. Bias was non-significantly different from zero. Thick horizontal line shows $\pm 10 \mathrm{~mm} \mathrm{Hg}$ distance from bias. 
A

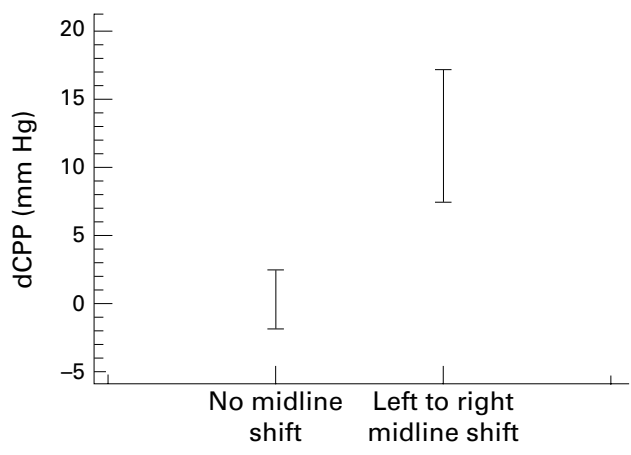

B

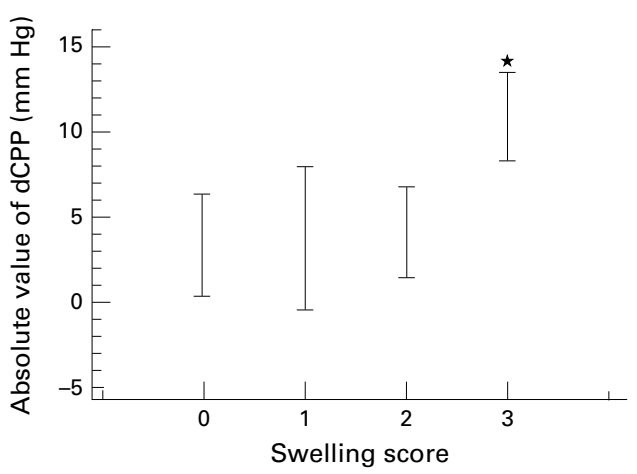

C

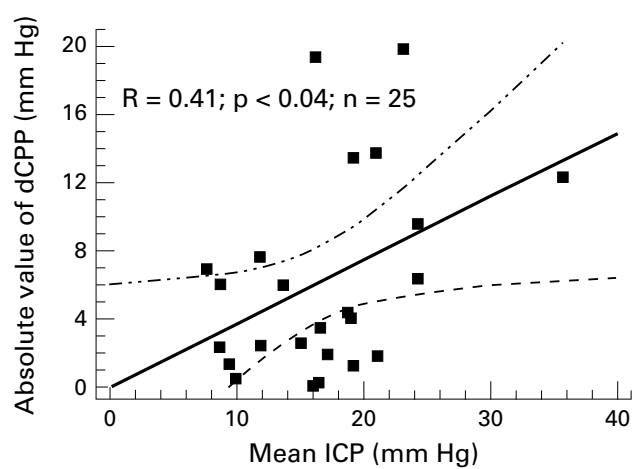

Figure 3 Statistical analysis of $d C P P(d C P P=$ left side $n C P P$ - right side $n C P P ; n=25$ patients). (A) $d C P P$ is significantly positive in patients with left to right midline shift $(p<0.021 ;$ ANOVA; $F$ test). (B) absolute value of $d C P P$ is significantly increased in patients with the highest swelling score $(p<0.026$, ANOVA; $F$ test $)$. (C) absolute value of CPP increases when measured mean ICP increases $(p<0.04$, regression analysis).

A typical plateau wave was recorded on day 5, with a slight and symmetric drop in FV and good pattern of accordance between CPP and nCPP.

Recordings performed on days 10 and 11 illustrate the clinical significance of transcranial Doppler in the assessment of cerebrovascular haemodynamics (see details on fig 5). During the night between days 9 and 10, the ICP signal decreased significantly to a very low value with a poor pulsatility and CPP increased accordingly. This was caused by ICP transducer malfunction. On day 10 (fig 5) the CPP and $\mathrm{nCPP}$ were obviously different, due to faulty readings of the ICP. At this stage the mean FV decreased and nCPP dropped reaching an extremely low value (less than $20 \mathrm{~mm}$ $\mathrm{Hg}$ ), consistent with a plateau wave.
The data illustrating changes in the FV pulsatility pattern are presented in fig 5 . At the beginning of the recording the diastolic FV was normal. It then fell steeply towards 0 during the first 3 minutes of the recording. This pattern is characteristic of a plateau wave of $\mathrm{ICP}^{13}$ which has been detected using $\mathrm{nCPP}$ in an absence of direct CPP recording.

When, on day 11, a new ICP transducer had been inserted, the measured CPP was close to the $\mathrm{nCPP}$ recorded on the previous day at the baseline.

\section{Discussion}

In a broad sense, the CPP reflects the physiological conditions for blood flow. The perfusion pressure of any organ is defined as the arithmetic difference between the mean inflow and outflow pressures.

In the brain, the cerebral blood inflow pressure is considered to be close to the mean ABP. The cerebral blood outflow pressure is not far from the pressure in the bridging veins, which is near the mean ICP. Hence, the mean CPP can be estimated with the formula:

\section{$\mathrm{CPP}=$ mean $\mathrm{ABP}-$ mean $\mathrm{ICP}$}

In clinical practice both variables ICP and ABP can be measured with limited accuracy. The case illustrated in fig 5 is a good example of the problems due to displacement of ICP microtransducer.

At the bedside, ICP measurements are normally confined to one hemisphere only, therefore any intracranial pressure gradients cannot be routinely monitored. Drift of ICP transducers ${ }^{14}$ can also be a source of gross misinterpretations. The measurement of $\mathrm{ABP}$ from the radial or dorsalis pedis arteries is not always representative for arterial pressure in the basal brain arteries, particularly under stress or in the presence of continuous infusion of active vasopresors.

\section{CPP: NOT A NUMBER BUT A CONDITION FOR} CEREBRAL BLOOD TO FLOW

In clinical practice, brain perfusion is better represented by global $\mathrm{CBF}$, rather than by a numerical value of CPP. Therefore, monitoring of CBF with all its heterogeneity (both spatial and temporal) would seem more appropriate. However, at the present stage of medical technology, there is no method for continuous CBF monitoring available. Of course, MRI or PET would provide the best accuracy and spatial resolutions but their availability at the bedside is limited..$^{15}$ Laser Doppler flowmetry, and brain tissue biochemical monitoring, although promising, give only localised readings. ${ }^{16} 17$ Jugular blood oximetry is useful, but technical problems often reduce its reliability. Moreover, in the supine body position, a significant fraction of the venous drainage from the brain takes place through the prevertebral venous plexus, rather than the jugular veins.

The use of transcranial Doppler flowmetry has many advantages. It is non-invasive and repeatable, signals may be obtained from different arteries at the same time, and continuous monitoring is possible with more 


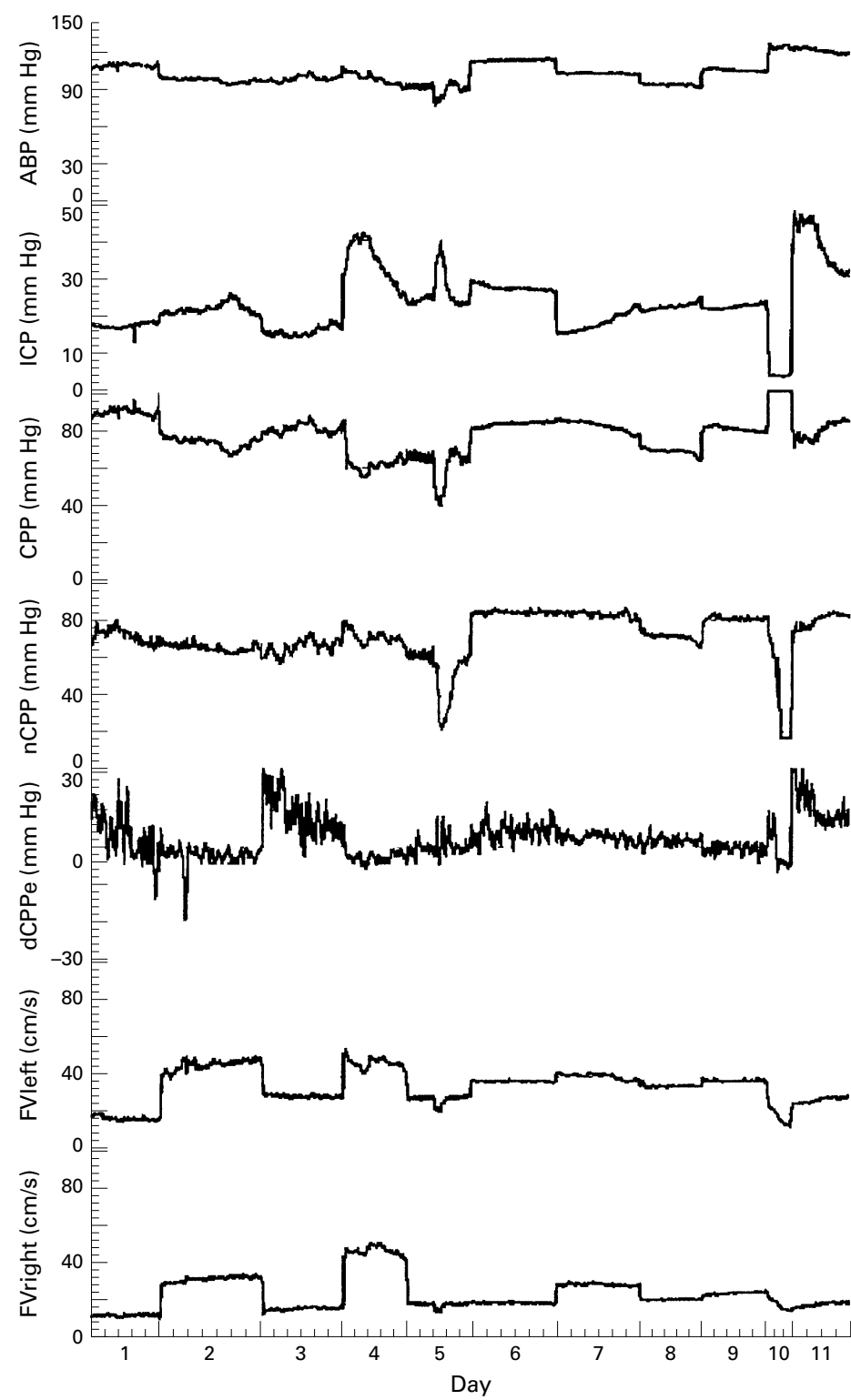

Figure 4 Daily recordings of mean ICP, arterial pressure (ABP), cerebral perfusion pressure $(C P P=A B P-I C P)$, left and right mean flow velocity ( $F V$ left and $F V$ right), non-invasive CPP ( $n C P P)$, and left to right difference in $n C P P(d C P P)$ in a patient after head injury. Signals were recorded for at least 30 minutes each day.

efficient bilateral probe holders and with the advent of autofocusing piezo-array ultrasound transducers glued to the scalp.

The problem lies in how to interpret blood flow velocity as a volume flow. Some attempts, although only partially successful, have been made using a specific analysis of Doppler spectra. ${ }^{18}$ The most promising approach to monitor brain perfusion seems to be to use the distinctive features of the blood flow velocity waveform. Many authors have pointed out that either an increase in transcranial Doppler pulsatility, ${ }^{9}{ }^{19}$ diminishing diastolic flow velocity, ${ }^{20}{ }^{21}$ or a decrease of the ratio of diastolic to mean flow velocity ${ }^{10}$ reliably indicate decreased brain perfusion. Therefore, transcranial Doppler waveform can be "translated" to brain perfusion continuously. This translation does not necessarily need to be done on the "'number to number" basis. In fact, in clinical practice the most often asked question is whether perfusion is adequate or not adequate.

On the other hand, almost all therapy protocols are based on CPP as a number. This was a reason why the formula: $\mathrm{ABP} \times \mathrm{FVd} / \mathrm{FVm}$, was initially proposed. ${ }^{10}$

ACCURACY OF ESTIMATION OF CPP VIA nCPP Both $\mathrm{CPP}=\mathrm{ABP}-\mathrm{ICP}$ and $\mathrm{nCPP}$ can be calculated with a limited accuracy and both are based on different physiological models which may not always be valid. Nevertheless, CPP calculated as the difference between ABP and ICP has become a gold standard with which an nCPP should be compared.

In our trial, the overall accuracy of this nCPP estimation was surprisingly good. The 95\% confidence interval for predictor was more than $50 \%$ smaller than reported using previous methods. ${ }^{68}$ The clinician may expect that in $80 \%$ of cases the error will be less than $10 \mathrm{~mm}$ $\mathrm{Hg}$ and in $90 \%$ of cases less than $13 \mathrm{~mm} \mathrm{Hg}$. Apart from a value of nCPP, the conditions for brain perfusion can be visually assessed from the transcranial Doppler waveform. Confounding factors in the estimation of CPP using nCPP have been identified previously. ${ }^{10}$ These include changes in arterial blood $\mathrm{CO}_{2}$ concentration, hyperaemia, and probably vasospasm. Our study confirmed previous findings ${ }^{10}$ that a non-invasive technique to measure CPP can reliably detect dynamic phenomena such as " $B$ waves" and plateau waves or other transitional changes in CPP.

SIDE TO SIDE DIFFERENCE IN nCPP

First attempts to demonstrate interhemispheric pressure gradients in the $1990 \mathrm{~s},{ }^{22}$ all indicated poor correlation between side to side asymmetry based on CT and invasive ICP differences. Continuous measurement of bilateral nCPP may be useful in clinical practice in future, but the validity of this new parameter should be investigated thoroughly beforehand. Although our group analysis was limited $(n=25)$ there was early evidence of clinical significance of side to side difference in $\mathrm{nCPP}$ ( $d C P P=$ left $\mathrm{nCPP}-$ right $\mathrm{nCPP}$ ).

Firstly, dCPP obviously depends on how accurately the MCAs are insonated. Side to side differences in the angle of insonation are not crucial as the ratio of $\mathrm{FVd} / \mathrm{FVm}$ does not depend on angle of insonation (providing the angle is not larger than $30^{\circ}$ ). However, when mounting ultrasound probes on a patient's head, care should be taken to optimise symmetry of the flow velocity readings on the screen.

The absolute value of dCPP increased significantly with increased mean ICP. Similar findings have been published in a report ${ }^{23}$ on bilateral jugular venous oxygen concentrations after head injury: side to side differences in $\mathrm{sJO}_{2}$ increased with intracranial hypertension.

The absolute value of dCPP correlated with our simplified brain swelling score: in severe swelling (score $=3$ ) the value of averaged side to side differences was significantly larger (fig $3 \mathrm{C})$ than in lower swelling score groups. 


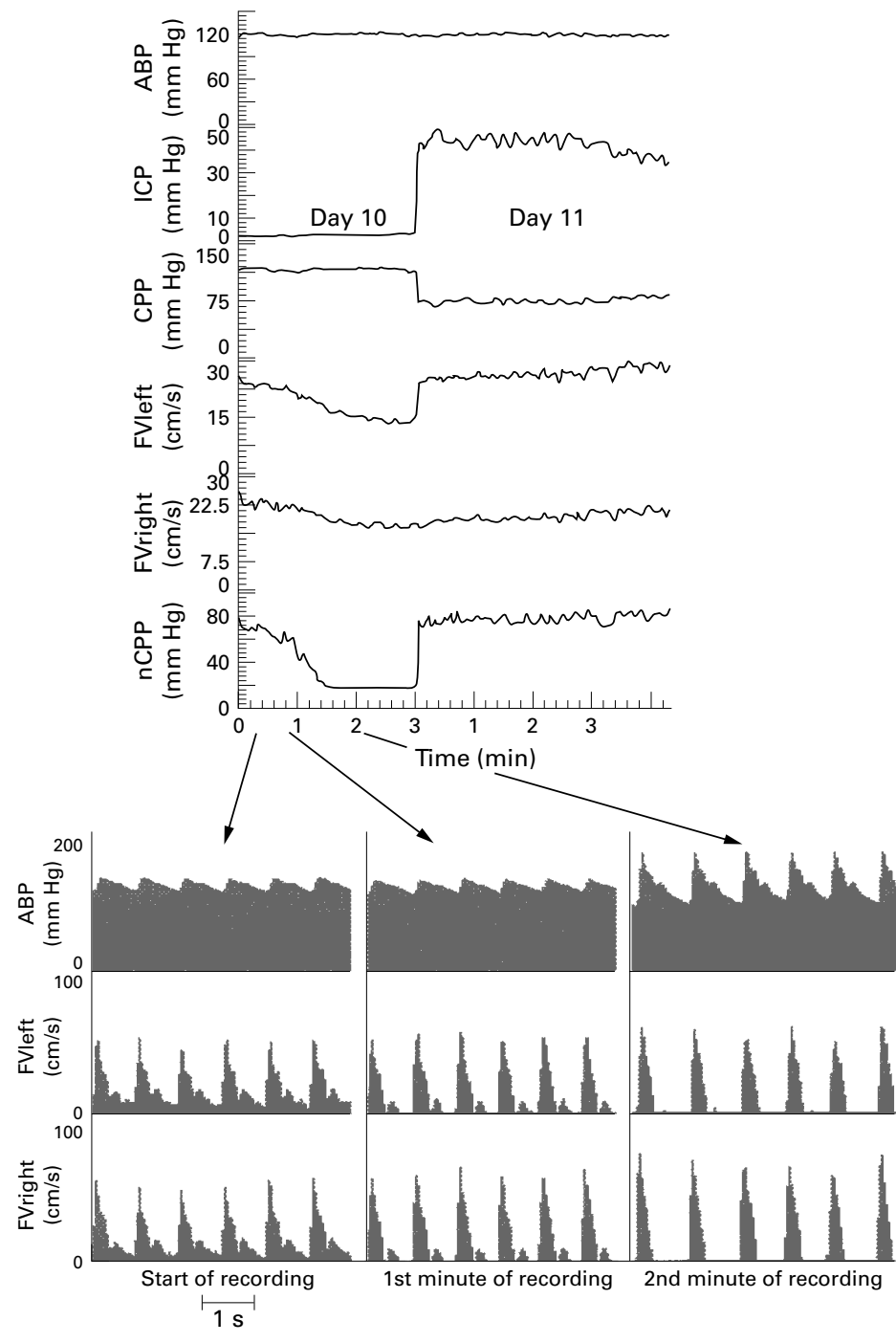

Figure 5 Recording made on days 10 and 11 when the invasive ICP transducer was defective. CPP was obviously artefactual. nCPP demonstrated a deep decrease during the recording, which coincided with a characteristic change in flow velocity waveform (below) with normal (left graph-beginning of recording); diminishing (1st minute of recording) and absent diastolic blood flow (2nd minute of recording). On day 11 a new ICP sensor was inserted and the CPP was again close to $n C P P$.

The number of patients with midline shift was small $(n=4)$, therefore our findings concerning dCPP can be only interpreted as anecdotal. Nevertheless, in all four patients nCPP was significantly greater in the swollen hemisphere. Conventional wisdom says the opposite: if ABP on both sides is the same, and ICP on the "expanding" side is greater, ${ }^{22}$ a lower CPP would be expected on this side. Our finding of an increased nCPP in the swollen hemisphere can be explained by relative hyperaemia, perhaps associated with ipsilateral vasogenic oedema. This hypothesis can be supported by findings on bilateral ultrasongraphy after brain trauma, ${ }^{24}$ indicating that autoregulation of $\mathrm{CBF}$ is significantly impaired on the expanding side of the brain. Failure of autoregulation at an early stage after brain trauma is commonly caused by vasoplegia leading to a decrease of cerebrovascular resistance and functional hyperaemia (hyperperfusion associated with a greater than expected value of $\mathrm{nCPP}$ ) on this side of the brain.
Indeed, in our patients absolute blood flow velocity was significantly higher in the swollen hemisphere $(\mathrm{p}<0.05 ; \mathrm{n}=4)$. Nevertheless, these early hypotheses and the real nature of dCPP need further clinical investigation.

\section{Conclusion}

The prototype transcranial Doppler based device for non-invasive bilateral monitoring of CPP showed satisfactory accordance with direct CPP measurements in a range from 60 to $100 \mathrm{~mm} \mathrm{Hg}$. The major advantage of the method lies in the ability to monitor patients in whom insertion of an ICP transducer cannot by justified and to detect whether CPP decreases, increases, or stays constant in a given case over time.

EAS received a scholarship from the University of ClermontFerrand, France. MC and SKP are on leave from Warsaw University of Technology, Poland. SKP received the "Fees Studentversity of Technology, Poland. SKP received the "Fees StudentTrust, the "Overseas Research Studentship" awarded by the Trust, the "Overseas Research Studentship" awarded by the
Committee of Vice-Chancellors and Principals of the Universities of the UK. MC acts as a consultant to Deltex, Chichester, UK.

1 Miller JD, Stanek A, Langfitt TW. Concepts of cerebral perfusion pressure and vascular compression during intracranial hypertension. Prog Brain Res 1972;35:411-32.

2 Jones PA, Andrews PJ, Midgley S, et al. Measuring the burden of secondary insults in head-injured patients. f Neurosurg Anesthesiol 1994;6:4-14.

3 Marmarou A, Anderson RL, Ward JD, et al. Impact of ICP instability and hypotension on outcome in patients with severe head trauma. I Neurosurg 1991:75(suppl): W559-66.

4 Rosner MJ, Rosner SD, Johnson AH. Cerebral perfusion pressure: management protocol and clinical results. $\mathcal{F} \mathrm{Neu}$ rosurg 1995;83:949-62

5 Czosnyka M, Smielewski P, Piechnik S, et al. Continuous assessment of cerebral autoregulation: clinical verification of the method in head injured patients. $f$ Cerebral Blood Flow Metab 1999;19:S362.

6 Al Rawi PG, Smielewski P, Kirkpatrick PJ. Preliminary evaluation of a prototype spatial resolved spectrometer. Acta Neurochir Suppl (Wien) 1997;71:255-7.

7 Dahl A, Lindegaard KF, Rusell D, et al. A comparison of transcranial Doppler and cerebral blood flow studies to assess cerebral vasoreactivity. Stroke 1992;23:15-19.

8 Aaslid R, Lundar T, Lindegaard K-F, et al. Estimation of cerebral perfusion pressure from arterial blood pressure and transcranial Doppler recordings. In: Miller JD, Teasdale GM, Rowan JO, et al, eds. Intracranial pressure VI. Berlin: Springer Verlag, 1986:229-31.

9 Chan KH, Miller DJ, Dearden M, et al. The effect of changes in cerebral perfusion pressure upon middle cerebral artery blood flow velocity and jugular bulb venous oxygen saturation after severe brain trauma. 7 Neurosurg 1992;77:55-61

10 Czosnyka M, Matta BF, Smielewski P, et al. Cerebral perfusion pressure in head-injured patients: a non-invasive assessment using transcranial Doppler ultrasonography. $\mathcal{F}$ Neurosurg 1998;88:802-8.

11 Marshall LF, Bowers-Marshall S, Klauber MR, et al. A new classification of head injury based on computerized tomography. F Neurosurg 1991;75(suppl):S14-S20.

12 Bland JM, Altman DG. Statistical methods for assessing agreement between two methods of clinical measurement. Lancet 1986:i:307-10.

13 Czosnyka M, Smielewski P, Piechnik S, et al. Hemodynamic characterization of intracranial pressure plateau waves in characterization of intracranial pressure plateau wav

14 Robertson CS, Narayan RK, Contant CF, et al. Clinical experience with a continuous monitor of intracranial comexperience with a continuous monitor
pliance. J Neurosurg 1989;71:673-80.

15 Bergsneider M, Hovda DA, Shalmon E, et al. Cerebral hyperglycolysis following severe traumatic brain injury in humans: a positron emission tomography study. $\mathcal{f}$ Neurosurg 1997;86:241-51.

16 Valadka AB, Gopinath SP, Contant CF, et al. Relationship of brain tissue $\mathrm{PO}_{2}$ to outcome after severe head injury. Crit Care Med 1998;26:1576-81.

17 Vespa P, Prins M, Ronne-Engstrom E, et al. Increase in extracellular glutamate caused by reduced cerebral perfusion pressure and seizures after human traumaticbrain injury: a microdialysis study f Neurosurg 1998:89:971-82.

8 Giller CA Hatab MR, Giller AM. Oscillations in cerebral bloo flow Cereb Blood Flow Metab 1999;19:452-9.

19 Klingelhofer J, Conrad B, Benecke R, et al. Evaluation of intracranial pressure from transcranial Doppler studies in intracranial pressure from transcranial Doppl
cerebral disease. $\mathcal{F}$ Neurol 1988;235:159-62. 
20 Nagai H, Moritake K, Takaya M. Correlation between transcranial Doppler ultrasonography and regional cerebral blood flow in experimental intracranial hypertension [discussion]. Stroke 1997;28:603-8.

21 Zurynski Y, Dorsch N, Pearson I, et al. Transcrania Doppler ultrasound in brain death: experience in 140 patients. Neurol Res 1991;13:248-52.

22 Wolfla CE, Luerssen TG, Bowman RM. Regional brain tissue pressure gradients created by expanding extradural temporal mass lesion. $\mathcal{F}$ Neurosurg 1997;86:505-10.

23 Metz C, Holzschuh M, Bein T, et al. Monitoring of cerebral oxygen metabolism in the jugular bulb: reliability of unilateral measurements in severe head injury. $\mathcal{F}$ Cereb Blood Flow Metab 1998;18:332-43.

24 Schmidt EA, Czosnyka M, Seeley H, et al. Asymmetry of cerebral circulation following head injury. $\mathcal{F}$ Cerebral Blood Flow Metab 1999;19:S364. 